\title{
Cindy COIGNARD, Les Militantes du POUM, 1935-1980
}

Rennes, Presses universitaires de Rennes, coll. « Archives du féminisme », 2015, 250 p. Préface de Mercedes Yusta

\section{Karine Bergès}

\section{(2) OpenEdition}

1 Journals

Édition électronique

URL : http://journals.openedition.org/clio/13118

DOI : 10.4000/clio. 13118

ISSN : 1777-5299

\section{Éditeur}

Belin

Édition imprimée

Date de publication : 1 juin 2016

Pagination : 296-299

ISBN : 978-2-7011-9852-1

ISSN : 1252-7017

Référence électronique

Karine Bergès, "Cindy colgnard, Les Militantes du POUM, 1935-1980», Clio. Femmes, Genre, Histoire [En ligne], 43 | 2016, mis en ligne le 23 août 2016, consulté le 22 septembre 2020. URL : http:// journals.openedition.org/clio/13118; DOI : https://doi.org/10.4000/clio.13118

Ce document a été généré automatiquement le 22 septembre 2020.

Tous droits réservés 


\section{Cindy COIGNARD, Les Militantes du POUM, 1935-1980}

Rennes, Presses universitaires de Rennes, coll. « Archives du féminisme », 2015, 250 p. Préface de Mercedes Yusta

\section{Karine Bergès}

\section{RÉFÉRENCE}

Cindy COIGNARD, Les Militantes du POUM, 1935-1980, Rennes, Presses universitaires de Rennes, coll. « Archives du féminisme », 2015, 250 p. Préface de Mercedes Yusta

1 L'intérêt majeur de la thèse de Cindy Coignard dont cet ouvrage est issu est de combler un vide historiographique sur le militantisme des femmes marxistes au cours de la guerre civile espagnole (1936-1939). Hormis l'ouvrage pionnier de l'espagnole Mary Nash sur les femmes républicaines, publié en 1999 (Rojas: las mujeres republicanas en la guerra civil), il n'existait pas à ce jour de monographie sur l'histoire sociale et politique des femmes au sein du POUM (Parti ouvrier d'unification marxiste). Par un travail de recherche conséquent, l'auteure contribue ainsi à enrichir les travaux sur une histoire genrée de la guerre civile et à mieux saisir de nombreux pans de l'histoire sociale et politique espagnole.

2 Le terrain choisi est celui de la Catalogne des années trente, mais l'étude va bien audelà puisque l'on suit l'itinéraire des militantes en Espagne depuis la fondation du parti en 1935, sur les chemins de l'exil à partir de 1937, et jusqu'à sa dissolution officielle en 1980. Si comme l'indique très justement Mercedes Yusta dans la préface de l'ouvrage, « le POUM est un des grands perdants de la guerre civile, ses femmes sont les grandes oubliées de son histoire»(p.7), Cindy Coignard restitue l'histoire de ces militantes en mobilisant des sources écrites inédites mais surtout, et là réside à notre sens la force de cette recherche, en laissant la parole à ces grandes absentes de l'Histoire, dont les témoignages sont habilement insérés dans les trois grands chapitres qui structurent l'ouvrage. 
3 Le premier chapitre, qui porte sur la période antérieure à la guerre civile, contextualise «la construction politique d'une pensée politique » et interroge les lignes idéologiques du parti, à la fois dans un cadre espagnol et international, afin de clarifier les positions théoriques des idéologues du POUM à l'égard de la "question féminine » et l'héritage des théoriciens socialistes et communistes (Marx, Engels, Bebel, Lénine, Kollontaï). Dans une perspective de genre, les débats autour des rapports entre les sexes, du droit de vote, du divorce, de la sexualité, de la prostitution ou encore de l'exaltation de la figure de la mère-prolétaire ont le mérite de rendre compte de la difficile articulation entre la dialectique marxiste de la lutte des classes et l'émancipation des femmes, à l'œuvre dans les écrits des penseuses féministes espagnoles depuis la fin du XIX ${ }^{e}$ siècle. En outre, la compréhension des mécanismes de socialisation qui sous-tendent le processus d'engagement politique des militantes au sein du POUM vient confirmer que l'entrée des femmes dans le militantisme s'est opérée, sur la base d'une construction identitaire antérieure, au sein même de la structure familiale, pilier de la socialisation politique. Ainsi, ces jeunes femmes, issues essentiellement d'un milieu ouvrier urbain de gauche, adhèrent au parti par identification à un modèle familial masculin et, pour la plupart d'entre elles, ont en commun l'expérience du communisme libertaire de la révolution d'octobre 1934, qui forgera le socle de leur identité militante. L'analyse de leur engagement au prisme des travaux récents sur les cultures politiques en Espagne conduit à souligner l'aspect novateur de cette recherche, qui élabore une cartographie des trajectoires à la fois personnelles et collectives des militantes poumistes, comme en témoigne le deuxième chapitre de l'ouvrage.

Dédiées à "l'affirmation d'un militantisme féminin", ces pages retracent l'action militante des femmes du POUM au cours des années 1936 et 1937 et débutent par une sous-partie consacrée aux « images et représentations féminines en temps de guerre ». L'auteure y intègre un vaste corpus de sources inédites, notamment les articles de presse et les photographies d'époque, et, si elle met clairement en évidence l'héroïsation, voire la manipulation de l'image de la milicienne, le recours à ces outils de propagande au détriment de données plus objectives dessert quelque peu son ambition de reconstruire une histoire des femmes poumistes à partir de l'expérience du vécu. Cela dit, l'auteure accorde une large place à l'étude du Secrétariat Féminin du POUM qui encadre la mobilisation des Espagnoles, la participation des militantes aux activités politiques (meetings, propagande via la presse et la radio) et aux missions en temps de guerre: assistance et soins aux miliciens, accueil des réfugiés, approvisionnement $\mathrm{du}$ front, collectes de fond, sous l'égide du Secours Rouge International. Bien que ces activités témoignent de l'engagement total des militantes, elles sont toutefois révélatrices de la persistance d'activités genrées dans la répartition des tâches et de la reproduction des rôles différenciés, comme cela fut le cas dans les rangs de la plupart des organisations et partis politiques de gauche au cours des années trente. Résolument plus novatrices sont les initiatives entreprises au cours de la guerre civile pour éradiquer le fléau de la prostitution, aussi bien sur le front qu'à l'arrière, et pour repenser la réinsertion et la resocialisation des personnes prostituées. $\mathrm{Si}$ l'engagement de l'association anarchiste Mujeres Libres est avéré sur ces questions, l'étude de Cindy Coignard, et notamment son analyse des expérimentations locales menées dans la ville de Gérone, offre un cadre de réflexion plus large aux questionnements sur des thématiques en lien avec la sexualité et la centralité du corps, qui sont toujours au cœur des luttes féministes contemporaines. De la même façon, les dernières pages du chapitre apportent un éclairage nouveau sur ce qui fait la spécificité 
du POUM : la défense de l'éducation en tant qu'instrument-clé dans l'émancipation de la classe des travailleurs. Avec le souci de recontextualiser les innovations éducatives, dans le sillage des théories des pédagogues de l'École moderne tels qu'A. Ferrière, O. Decroly, C. Freinet, M. Montessori et F. Ferrer i Guardia ou de l'ILE (Institución Libre de Enseñanza, 1876), l'auteure revient longuement sur la généalogie de cette rénovation pédagogique en Espagne depuis le $\mathrm{XIX}^{\mathrm{e}}$ siècle et démontre la spécificité d'un engagement genré des femmes du POUM au service de la modernisation de l'enseignement en Catalogne.

5 Le dernier chapitre de l'ouvrage nous situe au-delà de la guerre civile et du terrain espagnol en se focalisant sur l'expérience des femmes du POUM en exil (France, Cuba, Venezuela, Argentine). À la suite des affrontements entre communistes et marxistes de mai 1937, à Barcelone, et de la déclaration de son illégalité le 15 juin 1937, « le parti entre dans une double clandestinité: par rapport au franquisme mais aussi au stalinisme» (p.167), ce qui a pour conséquence l'exil ou la détention de ses membres, dont l'analyse de la répression sexuée au sein de l'univers carcéral est ici particulièrement bien menée. L'auteure interroge ainsi l'évolution du militantisme des femmes poumistes en exil et développe la thèse d'une mutation de leur engagement: d'un «militantisme politique » en Espagne à un "militantisme de résistance » ou " de survie» en exil. À l'exception de la très populaire milicienne argentine Mika Etchebéhère, qui fut aux commandes d'une colonne motorisée du POUM pendant la guerre et dont la continuité de l'engagement antifasciste se lit dans les articles qu'elle rédige pour le journal Argentina Libre, les parcours des autres militantes en exil esquissent un «militantisme culturel » qui prend corps autour d'activités artistiques : la Nouvelle chanson catalane (Nova Cançó Catalana) pour la militante Teresa Rebull, l'écriture et la poésie surréaliste pour l'Anglo-Australienne Mary Low. Regroupé.e.s autour des Centres catalans (Casals), véritables noyaux de résistance et de transmission d'une culture catalane, les militant.e.s du POUM tentent de reconstruire leur culture politique hors des frontières espagnoles afin de surmonter le démembrement du parti et la crise identitaire liée au déracinement de la condition d'exilé. Tout aussi pertinentes, quoique plus succinctes, sont les dernières réflexions de Cindy Coignard autour des liens du Poumisme avec le féminisme entre 1970 et 1977, ainsi que les questionnements autour de la transmission mémorielle: ceux-ci démontrent que " chaque mémoire individuelle est un point de vue sur la mémoire collective » (p. 216) et donnent sens au témoignage qui revêt, plusieurs années après les faits relatés, une dimension militante. En définitive l'ouvrage de Cindy Coignard a le mérite, grâce à un remarquable travail de collecte de sources orales, de redonner la parole à des femmes qui n'ont pas eu le sentiment de "faire l'Histoire ", mais dont les mémoires ont permis d'élaborer à la fois une histoire sociale du militantisme féminin au sein du POUM, mais également une histoire politique du marxisme en Espagne. 


\section{AUTEURS}

\section{KARINE BERGÈS}

Université de Cergy-Pontoise

Laboratoire AGORA 